\title{
Histochemical and Biochemical Correlates of Ventilatory Muscle Fatigue in Emphysematous Hamsters
}

G. A. Farkas and Ch. Roussos

Meakins-Christie Laboratories, McGill University, Montreal, Quebec H3A $2 B 4$

A characteristics of the ve in control and elastase-induced emphysematous hamsters. The emphysematous group was divided into sedentary and endurance-trained groups. Endurance training consisted of treadmill running, $1 \mathrm{~h}$ a day, $7 \mathrm{~d}$ a week. The experimental period lasted $24 \mathrm{wk}$. Histochemically, the diaphragm from the sedentary emphysematous hamsters revealed a selective fast fiber atrophy which was prevented by endurance training. Training also led to a hypertrophy of the slow, high oxidative fibers. The external intercostals from both emphysematous groups revealed an increased proportion of fast oxidative fibers at the expense of a decreased number of fast glycolytic fibers. However, the fast fibers in both emphysematous groups were significantly atrophied as compared with controls. The internal intercostals revealed no adaptive changes in either size or proportion distribution of the various fiber types.

Biochemically, the diaphragm of the emphysematous animals had a significantly improved oxidative potential as measured by citrate synthase, and a reduced glycolytic capacity as indicated by phosphofructokinase activity, compared with controls. The magnitudes of the biochemical changes were similar in both emphysematous groups and were consistent for diaphragmatic samples taken from the costal and crural segments. The combined internal and external intercostals also underwent signif-

This work was previously published in abstract (1982. Fed. Proc. 41:1255).

Dr. Farkas's present address is the Pulmonary Division, University of Virginia Medical Center, Charlottesville, VA 22908. 1984.

Received for publication 31 March 1983 and in revised form 7 June

J. Clin. Invest.

(C) The American Society for Clinical Investigation, Inc. 0021-9738/84/10/1214/07 \$1.00

Volume 74, October 1984, 1214-1220 icant biochemical increases in their oxidative capacity. In addition, training of the emphysematous group led to an increased glycolytic potential of the intercostals.

\section{Introduction}

The ventilatory muscles adapt to either strength or endurance training, as do other skeletal muscles (1-3). In limb skeletal muscles, a repetitive low-tension type of exercise training, such as running, improves endurance and leads to an increase in oxidative capacity of the muscle $(4,5)$. We reported that the diaphragm of emphysematous hamsters was more resistant to fatigue than were control diaphragms (6), but the exact cause for the improved endurance was not apparent. We postulate that breathing in states of chronic emphysema is much like a form of high-repetitive, low-tension training stimulus. Therefore, we hypothesize that the ventilatory muscles of the emphysematous hamsters undergo cellular adaptations consistent with an improved oxidative profile.

Whole body exercise has been shown to improve the strength and endurance of the respiratory muscles in patients with cystic fibrosis (1). Similarly, the oxidative capacity of the diaphragm in rodents is markedly increased after an endurance training program (7-9). However, we found that the fatigability of the diaphragm was the same in trained emphysematous hamsters as in sedentary emphysematous hamsters (6). Therefore, we hypothesize that daily endurance training in the form of running does not induce biochemical alterations in the respiratory muscles of emphysematous animals over and above those occurring from the emphysematous condition.

\section{Methods}

To test these two hypotheses, the histochemical and biochemical properties of the respiratory muscles and the plantaris, a nonrespiratory control muscle, were measured in three groups of hamsters. 45 adult ( $>40 \mathrm{wk}$ ) male golden hamsters were randomly divided into control and emphysematous groups. Animals from both groups were anesthetized with an intraperitoneal injection of pentobarbital sodium $(6 \mathrm{mg} /$ $100 \mathrm{~g}$ body wt; Nembutal; Abbott Pharmaceuticals, Inc. Pharmaceutical Products Div., North Chicago, IL). Those animals composing the emphysematous groups were treated with a single endotracheal instil- 
lation of porcine pancreatic elastase, $0.2 \mathrm{mg}$ in $0.5 \mathrm{ml}$ of $0.15 \mathrm{M}$ $\mathrm{NaCl} / 100 \mathrm{~g}$ body wt (10). Control animals received a single endotracheal instillation of $0.5 \mathrm{ml}$ of $0.15 \mathrm{M} \mathrm{NaCl} / 100 \mathrm{~g}$ body wt. After a 3-wk recovery period, the emphysematous animals were randomly subdivided into a sedentary and an exercise group. The three experimental groups are referred to as sedentary control (SC), ${ }^{1}$ sedentary emphysematous (SE), and exercise emphysematous (EE). The exercise emphysematous group was subjected to a progressive training program of treadmill running. The training protocol has been previously published (6).

All studies were carried out $24 \mathrm{wk}$ after the endotracheal instillation of elastase or saline. Functional residual capacity, contractility, histochemistry, and biochemistry were measured in every animal. Their pulmonary and diaphragmatic contractile properties have been previously reported (6).

We investigated adaptations occurring in only those animals who successfully completed the entire experimental period. The only basis for rejecting animals from the sedentary groups was the death of an animal during the experimental period, whereas for the exercise emphysematous group, three successive days of noncompletion of the entire exercise period, due either to sickness or injured limbs, was also used as a basis for rejection. The possibility exists, therefore, that we may have inadvertently excluded or eliminated the most severely affected animals from the exercise emphysematous group, whereas they may have been included in the results of the sedentary emphysematous group.

Histochemical measurements. Muscle samples were removed from the anterior costal segment of the diaphragm, as well as from the internal and external intercostals of the second or third interspace, and thick cork sheet, supported with O.C.T. compound (Lab-Tek Products, Naperville, IL), and quickly immersed into isopentane cooled to its melting point with liquid nitrogen. Muscle samples were then stored at $-90^{\circ} \mathrm{C}$ until analyzed.

For the histochemical analyses, samples were removed from the freezer and equilibrated at $-20^{\circ} \mathrm{C}$ for $15-20 \mathrm{~min}$. The cork was secured to a cryostat block with O.C.T. compound. Serial sections 10 $\mu \mathrm{m}$ thick were cut, mounted on glass cover slides, and allowed to dry at room temperature. The sections were stained for alkaline dependent (pH 10.4) myofibrillar ATPase (11), NADH-diaphorase (12), and mitochondrial- $\alpha$ glycerophosphate dehydrogenase (13) activities.

Serial sections incubated for the various reactions were examined and photographed with a photomicroscope (Nikon Inc., Instrument Div., Garden City, NY). On the basis of their enzyme activities, fibers were classified by the criteria described by Peter et al. (14) as slow twitch oxidative (SO), fast twitch oxidative glycolytic (FOG), and fast twitch glycolytic (FG). The fibers, on Polaroid prints (Polaroid Corp., Cambridge, MA), were identified by their histochemical profile, and a particle size analyzer (Carl Zeiss, Inc., New York) was used to count the number of each fiber type and the diameter of each fiber. The cross-sectional area of each fiber type was calculated from the diameter. Approximately 150-200 different fibers were analyzed per print.

Biochemical measurements. To determine phosphofructokinase (PFK) and citrate synthase activity, homogenates of costal and crural diaphragm and the combined internal and external intercostal muscles

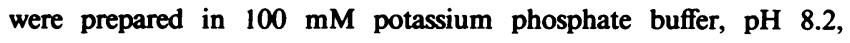

1. Abbreviations used in this paper: $\mathrm{EE}$, exercise emphysematous; FG, fast twitch glycolytic; FOG, fast twitch oxidative glycolytic; PFK, phosphofructokinase; SC, sedentary control; SE, sedentary emphysematous; SO, slow twitch oxidative. containing $10 \mathrm{mM}$ glutathione, $0.5 \mathrm{mM} \mathrm{ATP}, 5 \mathrm{mM} \mathrm{MgCl}$, and 30 $\mathrm{mM} \mathrm{NaF} \mathrm{(15).} \mathrm{Control} \mathrm{and} \mathrm{experimental} \mathrm{muscles} \mathrm{were} \mathrm{always} \mathrm{processed}$ and analyzed at the same time in a random order. PFK was measured spectrophotometrically at $37^{\circ} \mathrm{C}$ by the use of a coupling system as described by Mansour et al. (16). Citrate synthase was measured spectrophotometrically at $30^{\circ} \mathrm{C}$ by the method of Srere (17). Homogenates were frozen and thawed three times to disrupt the mitochondria. Enzyme activities of the homogenates were expressed in micromoles per minute per gram wet weight.

Statistical comparison was made between emphysematous groups and control groups by means of a $t$ test for unpaired samples (18), with significance set at $P<0.05$. Values are expressed as means \pm SEM.

\section{Results}

The initial and final body weights, as well as the final length in centimeters from the tip of the nose to the tip of the tail, are given in Table I. At the start of the experimental period, animals were assigned randomly to their respective groups, and body weight was virtually identical among the three groups. In the control animals, body weight remained constant throughout, indicating that the animals were full grown at the start of the study. By the completion of the experimental period, both emphysematous groups weighed $\sim 20 \%$ less than the control animals. Both groups of emphysematous animals had a twofold increase in functional residual capacity as compared with controls (6).

Muscle fiber type proportion and size. The results are summarized in Table II. There was no change in the fiber types in the diaphragm in either of the experimental groups. The FOG fibers from diaphragms of the sedentary emphysematous group were smaller than control FOG fibers. Training of the emphysematous animals prevented atrophy of the FOG fibers and also resulted in $22 \%$ hypertrophy of the diaphragmatic SO fibers as compared with control values (Table II).

Emphysema alone or in conjunction with training altered the fiber type population of the external intercostals so that the number of FOG fibers increased by $\sim 10 \%$ whereas the number of FG fibers decreased by $23 \%$ in both groups of emphysematous animals. The size of the external intercostal FG fibers also decreased by $15 \%$ in both groups of emphysematous hamsters (Table II). The size and number of external

Table I. Number of Animals, Initial and Final Body Weights, and Final Body Length of Hamsters from the Various Groups

\begin{tabular}{lllll}
\hline Group & $\begin{array}{l}\text { Final No. } \\
\text { of animals }\end{array}$ & $\begin{array}{l}\text { Initial body } \\
\text { weight }\end{array}$ & $\begin{array}{l}\text { Final body } \\
\text { weight }\end{array}$ & Length \\
\hline & & $g$ & $g$ & $\mathrm{~cm}$ \\
SC & 10 & $142.9 \pm 5.6$ & $146.0 \pm 3.6$ & $17.9 \pm 0.13$ \\
SE & 8 & $144.7 \pm 5.8$ & $112.5 \pm 2.7^{*}$ & $17.6 \pm 0.12$ \\
EE & 7 & $140.7 \pm 5.0$ & $118.2 \pm 5.4^{*}$ & $17.6 \pm 0.12$ \\
\hline
\end{tabular}

Values are means \pm SEM.

* Significantly different from SC group, $P<0.05$. 
Table II. Respiratory Muscle Fiber Types and Size from the Various Experimental Groups

\begin{tabular}{|c|c|c|c|c|c|c|}
\hline & \multicolumn{2}{|c|}{ Costal diaphragm } & \multicolumn{2}{|c|}{ External intercostals } & \multicolumn{2}{|c|}{ Internal intercostals } \\
\hline & No. & Size & No. & Size & No. & Size \\
\hline & $\%$ & $\mu m^{2}$ & $\%$ & $\mu m^{2}$ & $\%$ & $\mu m^{2}$ \\
\hline \multicolumn{7}{|l|}{ SC } \\
\hline SO & $23.2 \pm 1.0$ & $1,354.1 \pm 85.8$ & $18.0 \pm 3.1$ & $1,148.1 \pm 87.0$ & $16.9 \pm 2.3$ & $956.1 \pm 59.9$ \\
\hline FOG & $76.8 \pm 1.0$ & $1,627.3 \pm 65.4$ & $26.0 \pm 2.3$ & $1,380.1 \pm 92.6$ & $29.7 \pm 1.2$ & $1,217.9 \pm 81.0$ \\
\hline FG & - & - & $56.0 \pm 3.3$ & $1,572.5 \pm 82.2$ & $53.5 \pm 3.5$ & $1,558.8 \pm 84.7$ \\
\hline \multicolumn{7}{|l|}{ SE } \\
\hline SO & $25.3 \pm 2.0$ & $1,545.3 \pm 119.7$ & $19.3 \pm 5.4$ & $964.4 \pm 109.5$ & $19.3 \pm 3.5$ & $963.1 \pm 100.6$ \\
\hline FOG & $74.7 \pm 2.0$ & $1,115.2 \pm 119.6^{*}$ & $37.0 \pm 2.4$ & $1,232.9 \pm 67.1$ & $32.1 \pm 2.17$ & $1,240.1 \pm 101.6$ \\
\hline FG & - & - & $43.7 \pm 3.2^{*}$ & $1,329.7 \pm 65.2^{*}$ & $48.7 \pm 3.7$ & $1,428.8 \pm 111.1$ \\
\hline \multicolumn{7}{|l|}{ EE } \\
\hline SO & $25.5 \pm 1.1$ & $1,639.8 \pm 147.7^{*}$ & $18.8 \pm 2.4$ & $1,020.7 \pm 85.1$ & $20.1 \pm 4.5$ & $1,067.5 \pm 96.1$ \\
\hline FOG & $74.5 \pm 0.4$ & $1,539.6 \pm 148.9$ & $38.9 \pm 4.0^{*}$ & $1,045.4 \pm 65.2^{*}$ & $34.7 \pm 2.7$ & $1,228.4 \pm 161.7$ \\
\hline FG & - & - & $42.3 \pm 4.5^{*}$ & $1,350.0 \pm 94.48^{*}$ & $45.1 \pm 3.3$ & $1,814.6 \pm 214.3$ \\
\hline
\end{tabular}

Values are means \pm SEM. No. represents percentage of total fibers. * Significantly different from group SC, $P<0.05$.

intercostal SO fibers remained unchanged in both emphysematous groups. The internal intercostals revealed no histochemical evidence of fiber type alterations as a result of emphysema alone or with exercise training (Table II).

To establish whether weight loss per se had any effect on muscles, the proportion and size of muscle fiber types in the plantaris, a nonrespiratory muscle, were studied in both control and sedentary emphysematous animals. There was no change in either the proportion of the fiber type population or in the cross-sectional area of the various fiber types in the plantaris muscle (Table III) from sedentary control or emphysematous hamsters.

Muscle biochemistry. The diaphragms of both emphysematous groups showed significant changes consistent with increased oxidative and decreased glycolytic activity. Activity of the oxidative enzyme, citrate synthase, increased by $\sim 25 \%$, whereas activity of the glycolytic enzyme PFK decreased by $\sim 45 \%$ (Table IV). The results were similar in anterior costal and crural segments of diaphragm.

The intercostal muscles of both groups of emphysematous animals had a 55\% increase in citrate synthase activity (Table IV). Emphysema alone did not reduce intercostal muscle PFK activity, whereas exercise training superimposed on emphysema caused a $58 \%$ increase in intercostal muscle PFK activity (Table IV).

The citrate synthase values for the plantaris, a nonrespiratory muscle, for the three experimental groups were: SC, 34.1 \pm 2.3 ; $\mathrm{SE}, 37.0 \pm 2.9$; and $\mathrm{EE}, 48.7 \pm 2.8 \mu \mathrm{mol} / \mathrm{min}$ per $\mathrm{g}$. The values for the exercise emphysematous group were significantly increased as compared with either sedentary group. Thus, the exercise training program in the emphysematous hamsters produced alterations in the limb muscles consistent with a training effect.

\section{Discussion}

\section{Muscle fiber type proportion and size}

Diaphragm. We reported that the speed-related contractile twitch characteristics (namely, time to peak tension and onehalf relaxation time) of the diaphragm were not altered in emphysematous hamsters (6), suggesting that no major change

Table III. Plantaris Muscle Fiber Types and Size in Control and Emphysematous Hamsters

\begin{tabular}{llr}
\hline & \multicolumn{2}{l}{ Plantaris } \\
\cline { 2 - 3 } & No. & \multicolumn{1}{l}{ Size } \\
\hline & $\%$ & $\mu m^{2}$ \\
SC & & \\
SO & $13.6 \pm 1.4$ & $948.4 \pm 47.5$ \\
FOG & $39.8 \pm 3.2$ & $1,161.1 \pm 59.4$ \\
FG & $46.6 \pm 3.0$ & $1,612.9 \pm 79.2$ \\
SE & & \\
SO & $10.1 \pm 2.2$ & $1,029.6 \pm 117.4$ \\
FOG & $45.0 \pm 2.3$ & $1,319.4 \pm 149.5$ \\
FG & $44.9 \pm 3.2$ & $1,725.1 \pm 163.9$ \\
& &
\end{tabular}

Values are means \pm SEM. No. represents percentage of total fibers. No significant difference was noted between SC and SE groups. 
Table IV. Oxidative and Glycolytic Activity of the Various Groups' Ventilatory Muscles

\begin{tabular}{|c|c|c|c|c|c|c|}
\hline \multirow[b]{2}{*}{ Group } & \multicolumn{2}{|c|}{ Costal diaphragm } & \multicolumn{2}{|c|}{ Crural diaphragm } & \multicolumn{2}{|l|}{ Intercostals } \\
\hline & CS & PFK & CS & PFK & CS & PFK \\
\hline SC & $54.9 \pm 3.8$ & $45.1 \pm 6.4$ & $45.2 \pm 3.7$ & $25.8 \pm 5.6$ & $18.0 \pm 2.3$ & $22.6 \pm 6.1$ \\
\hline SE & $65.8 \pm 3.1^{*}$ & $25.6 \pm 6.4^{*}$ & $59.7 \pm 4.6^{*}$ & $11.7 \pm 3.1^{*}$ & $29.9 \pm 5.5^{*}$ & $30.6 \pm 4.1$ \\
\hline $\mathrm{EE}$ & $67.1 \pm 3.7^{*}$ & $25.3 \pm 3.6^{*}$ & $55.4 \pm 4.9^{*}$ & $14.3 \pm 1.8^{*}$ & $26.1 \pm 3.5^{*}$ & $35.6 \pm 3.9^{*}$ \\
\hline
\end{tabular}

Citrate synthase (CS) was measured at $30^{\circ} \mathrm{C}$. PFK was measured at $37^{\circ} \mathrm{C}$. Enzyme activity is expressed in micromoles of substrate converted per minute per gram of muscle wet weight. Values are means \pm SEM. * Significantly different from $\mathrm{SC}$ group, $P<0.05$.

in fiber distribution had occurred. The results of the present study indicate that this was indeed the case (Table II). A similar consistency of fiber type distribution in the hamster emphysema model has been previously reported by Kelsen et al. (19), and the proportion of fast to slow fibers in both hamster studies is similar. We found no FG fibers in our anterior costal diaphragm sections, whereas Kelsen et al. found that $45 \%$ of lateral costal diaphragm muscle fibers were FG. This discrepancy is probably due to the difference in sampling site. As shown by Riley and Berger (20), at least in the cat diaphragm, the anterior costal (sternal) diaphragm contains only high oxidative fibers, whereas in the lateral sections all three fiber types are found.

Keens et al. (21) reported that the diaphragm of rats can respond to a chronic ventilatory overload (tracheal banding) by increasing its percentage of SO fibers. In their study (21) the diaphragm had to overcome large airway resistances, whereas in the hamster model of emphysema, airway resistance is unchanged (22). Thus, the difference in the respiratory load on the diaphragm probably accounts for the difference in the histochemical response between the present study and that of Keens et al. (21).

Usually, conversion of fiber types from fast to slow or from slow to fast does not occur with physical training $(4,8)$. Lieberman et al. (8) reported that training in guinea pig diaphragm led to an increased number of high oxidative fibers but gave no evidence of true fiber-type transformation. In the hamster diaphragm, at least in the section we tested, the fibers are $100 \%$ high oxidative (Table II), so that any increase in the relative oxidative capacity of the individual fibers would not be detected histochemically; FOG fibers in the experimental animals remained FOG fibers but with an increased oxidative capacity as compared with control.

We found that emphysema alone caused FOG fibers in the diaphragm to atrophy, whereas the combination of emphysema and exercise training prevented atrophy of FOG fibers and caused SO fibers to hypertrophy. In contrast, Kelsen et al. (19) found that all types of diaphragmatic fibers hypertrophied in sedentary emphysematous hamsters. These differences may be accounted for by the experimental conditions. Although the basic hamster emphysema model was the same in both studies,
Kelsen's animals were studied 12 wk after elastase instillation (19), whereas ours were studied at 24 wk. Our hamster lung volumes were substantially larger, total lung capacity being increased by $125 \%$ (6) as compared with $60 \%$ in Kelsen's hamsters (19). This is consistent with the observations of Snider and Sherter (10) that lung volume increases progressively for 6 mo after a single instillation of elastase. Moreover, at the time of study our animals weighed less than control animals, whereas in the study of Kelsen et al. (19) body weight was the same in both control and emphysematous animals. We selected the longer duration after elastase instillation to examine the chronic effects of emphysema on the respiratory muscles over a period that represents a third of the normal hamster life span.

Selective fast fiber atrophy has been shown to result from stress overload (23). However, it is unlikely that the FOG fiber atrophy seen in diaphragms of our emphysematous animals can be attributed to overload, because FOG fiber atrophy did not occur in emphysematous animals that also underwent exercise training. Exercise increases respiratory rate, tidal volume, and minute ventilation. It is possible that the exercise ventilatory pattern increased fast fiber recruitment and thus prevented atrophy.

Exercise training also caused SO fibers to hypertrophy. Similar results were obtained in exercise trained guinea pigs (8) and in rats subjected to tracheal banding (21). However, in the latter experiments the rats were immature, and the increase in SO fiber diameter was apparent only when fiber size was corrected for diaphragm weight in order to take into account growth of animals during the period of observation. In the present study, the animals were mature, and diaphragms were not weighed. Thus, direct comparisons with the trachealbanded rats are not possible. Moreover, since diaphragm muscle fibers from emphysematous hamsters are shorter than control, owing to a loss of sarcomeres $(6,19,24)$, correction of fiber size on the basis of diaphragm weight would tend to overestimate the fiber diameter or cross-sectional area.

Studies of respiratory muscles from human chronic obstructive lung disease have yielded conflicting results. Measurements of diaphragm fibers at necropsy showed hypertrophy, as judged from increases in diaphragm muscle fiber diameter 
(25) or cross-sectional area (26). However, the antemortum clinical and nutritional status of control and emphysematous patients was not given, the number of subjects was small (25), and the morphologic severity of emphysema was pronounced in only 6 of 18 patients considered by Scott and Hoy to have emphysema (26). Two recent studies, based on respiratory muscle biopsy samples obtained at thoracotomy, showed atrophy rather than hypertrophy. Sanchez and co-workers (27) found atrophy of both Type I (SO) and Type II (FOG and FG) fibers for the diaphragm, whereas Hughes et al. (28) found modest atrophy of Type II fibers in diaphragm, although the body weight was normal $(27,28)$.

Intercostals. As the efficiency of the diaphragm is progressively decreased with pulmonary hyperinflation, the external intercostals should be recruited more in order to maintain ventilation. In both emphysematous groups, the external intercostals responded by an increase in the FOG fiber population at the expense of the FG fibers (Table II). The increase in the number of FOG fibers is consistent with the increased oxidative capacity of the intercostals in emphysema (Table IV). In addition to being reduced in number, fast fibers in both emphysematous groups were smaller in size as compared with fast fibers of the control group (Table II). Faulkner et al. (23) previously noted decreases in cross-sectional area in fast fibers of plantaris muscle after a training regime, but total muscle cross-sectional area was unchanged.

Keens et al. (21) reported that tracheal banding resulted in severe alterations in the internal intercostals (increased FOG and decreased number of FG fibers) with no changes occurring in the external intercostals. Similarly, in human chronic obstructive pulmonary disease $(28,29)$ it has also been noted that the internal intercostals are more severely affected than the external intercostals, with the atrophy of type II fibers being related to the severity of the airflow obstruction. In emphysematous hamsters, Park et al. (22) have shown that the airway resistance is unaffected, whereas in the tracheal banded animals (21) and patients (29) both inspiratory and expiratory resistance were probably increased.

The adaptations noted above in intercostal muscle histochemical characteristics are consistent with reduced susceptibility to fatigue in both groups of emphysematous animals as compared with normal animals. Since changes in the sedentary and trained emphysematous animals were usually of the same magnitude, their resistance to fatigue was probably the same.

\section{Muscle biochemical profile}

Diaphragm. The observed increase in citrate synthase activity (Table IV) is consistent with an increase in overall oxidative capacity (30). It is also consistent with our findings of reduced fatigability (6) in diaphragms from both groups of emphysematous animals. The extent to which adaptations occur in a muscle depends not only on the alterations in its function, but also on the intensity and duration of the stress $(15,30)$. Therefore, the intensity and/or duration of the $1 \mathrm{~h}$ daily exercise training period was probably not enough to produce additional increases in oxidative capacity beyond those occurring with the chronic 24-h-a-day load imposed by emphysema.

In response to increased use, exercise-induced decreases in PFK activity in FOG fibers are thought to be obscured by increases in PFK activity occurring in SO fibers and by the unchanged PFK activity occurring in FG fibers (5). However, as noted in Table IV, the anterior costal diaphragm of the hamster is composed primarily (80\%) of FOG fibers, so that a decrease in PFK activity in the diaphragm with emphysema or training (Table IV) is not unexpected. Endurance training in the present study did not further decrease the diaphragmatic PFK values (Table IV), tending to substantiate the view that daily exercise of $1 \mathrm{~h}$ in the emphysematous animals was indeed too short to cause any further biochemical changes. Alternatively, the adaptations induced by the emphysematous condition may have been enough to accommodate the added stress of 1 $h$ of daily exercise. Ianuzzo et al. (7) showed that diaphragmatic PFK activity increased in normal rats after a period of training, but the difference in fiber population of rat diaphragm (34\% SO, 38\% FOG, 28\% FG) compared with hamster diaphragm could account for different PFK responses to enhanced activity.

An increase in intramuscular energy stores can also increase endurance. In a complementary project, we noted that the intramuscular glycogen level in the diaphragm of sedentary emphysematous animals was significantly higher than values obtained from control animals (unpublished observations). The effects of exercise training in emphysematous hamsters on the glycogen content of the diaphragm were not investigated. Therefore, although not measured in the present study, part of the increased endurance of the diaphragm of the emphysematous animals (6) may have also been due to a higher initial intradiaphragmatic glycogen level.

Intercostals. As the efficiency of the diaphragm is reduced with hyperinflation (3i), a greater dependency on the intercostals or the accessory muscles of inspiration (or both) to produce expansion of the rib cage is expected. We could not measure the in vitro fatigability of the intercostals owing to their small size and the limitations of our recording system. If the intercostals are recruited to a greater extent in the emphysematous hamster, these muscles should undergo biochemical alterations consistent with increased endurance (reduced fatigability). The intercostal muscles from emphysematous hamsters did increase their citrate synthase activity (Table IV), consistent with the results of Keens et al. in tracheal-banded rats (21).

Physical exercise, in which high levels of ventilation are reached and maintained for prolonged periods, might also increase the oxidative capacity of the intercostals. However, Moore and Gollnick (9) reported that rats undergoing 8-26 wk of treadmill running did not have increases in intercostal muscle oxidative capacity. These animals were breathing at normal lung volume. In the present study, training of emphysematous animals did not further increase intercostal muscle citrate synthase activity beyond that seen with emphysema alone (Table IV). Thus, the duration or the intensity of the training period, in relation to the load produced by the 
emphysema, appears to have been either too brief or too small to further the adaptations occurring in response to emphysema per se. Moreover, as previously mentioned, we may have selectively eliminated those animals from the training group that were the most severely affected and possibly the most dependent on their intercostals in order to ventilate.

Keens et al. (21) reported that chronic tracheal banding did not lead to changes in the PFK activity of the intercostals in rats, which is in agreement with our results from sedentary and exercised emphysematous animals (Table IV). Training has been previously associated with increases in PFK activity in rat soleus (32) and rat diaphragm (7).

Goldspink (33) reported that in states of undernutrition leading to a $20 \%$ loss in body weight, fast muscle fibers of the biceps brachii and of the soleus were more severely atrophied than slow fibers. In order to account for the effects of $20 \%$ weight loss in the present study, histochemical and biochemical measurements were evaluated on the plantaris from control and elastase-treated animals. The decrease in body weight observed in the emphysematous animals (Table I) did not produce any alterations in the size or distribution of the fiber types (Table III) or in the level of key oxidative or glycolytic enzymes of the plantaris. These results are consistent with our previous report (6), which showed no difference in the in vitro fatigability among the plantaris from control and elastasetreated animals. Thus, alterations noted in the ventilatory muscles are most probably caused by alterations in function due to the emphysema.

Based on the above, and from previous work (19), the respiratory muscles or primarily the diaphragm, appear to adapt to chronic hyperinflation in the following sequence. When the hyperinflation is moderate, the diaphragm adapts mainly by increasing its fiber size. Since the maximal tension a muscle generates is related to its cross-sectional size (34), maximal inspiratory pressures can be maintained even though the diaphragm may be operating at somewhat of a mechanical or geometric disadvantage. As the disease progresses and hyperinflation is severe, atrophy of the diaphragmatic fibers occurs, possible explaining the poor pressure generated by many emphysematous patients (35). It would also appear that endurance exercise prevents the atrophy from occurring in the late stages and thus could help maintain the strength-generating capabilities of the respiratory muscles.

\section{Acknowledgments}

The authors thank Ms. K. Gardiner for her technical assistance, and Drs. P. Gardiner and A. W. Taylor, University of Montreal, and Dr. C. D. Ianuzzo, York University (Toronto), for their helpful comments and advice throughout this study and the preparation of this manuscript.

This work was supported by the Medical Research Council of Canada and the Quebec Thoracic Society.

\section{References}

1. Keens, T. G., I. R. B. Krastins, E. M. Wannamaker, H. Levison, D. N. Crozier, and A. C. Bryan. 1977. Ventilatory muscle endurance training in normal subjects and patients with cystic fibrosis. Am. Rev. Respir. Dis. 116:853-860.

2. Leith, D. E., and M. Bradley. 1976. Ventilatory muscle strength and endurance training. J. Appl. Physiol. 41:508-516.

3. Bradley, M. E., and D. E. Leith. 1978. Ventilatory muscle training and the oxygen cost of sustained hypercapnea. J. Appl. Physiol. Respir. Environ. Exercise Physiol. 45:885-892.

4. Barnard, R. J., V. R. Edgerton, and J. B. Peter. 1970. Effect of exercise on skeletal muscle. I. Biochemical and histochemical properties. J. Appl. Physiol. 28:762-766.

5. Baldwin, K., and W. W. Winder. 1977. Adaptive responses in different types of muscle fibers to endurance exercise. Ann. NY Acad. Sci. 301:411-423.

6. Farkas, G. A., and Ch. Roussos. 1982. Adaptability of the hamster diaphragm to exercise and/or emphysema. J. Appl. Physiol. Respir. Environ. Exercise Physiol. 53:1263-1272.

7. Ianuzzo, C. D., E. G. Noble, N. Hamilton, and B. Dabrowski. 1982. Effects of streptozotocin diabetes, insulin treatment and training on the diaphragm. J. Appl. Physiol. Respir. Environ. Exercise Physiol. 52:1471-1475.

8. Lieberman, D. A., L. C. Maxwell, and J. A. Faulkner. 1972. Adaptation of guinea pig diaphragm muscle to aging and endurance training. Am. J. Physiol. 222:556-560.

9. Moore, R. L., and P. D. Gollnick. 1982. Response of ventilatory muscle of the rat to endurance training. Pfleugers Arch. Eur. J. Physiol. 392:268-271.

10. Snider, G. L., and C. B. Sherter. 1977. A one-year study of the evolution of elastase induced emphysema in hamsters. J. Appl. Physiol. Respir. Environ. Exercise Physiol. 43:721-729.

11. Guth, L., and F. Samaha. 1969. Qualitative differences between actomyosin ATPase of slow and fast mammalian muscle. Exp. Neurol. 25:138-152.

12. Peter, J., D. Verhaag, and M. Worsfold. 1970. Studies of steroid myopathy. Examination of the possible effect of triamcinolone on mitochondria and sarcotubular vesicles of rat skeletal muscle. Biochem. Pharmacol. 19:1627-1636.

13. Wattenberg, L., and J. Leong. 1960. Effects of coenzyme $Q_{10}$ and menadione on succinate dehydrogenase activity as measured by tetrazolium salt reaction. J. Histochem. Cytochem. 8:269-303.

14. Peter, J., R. Barnard, V. R. Edgerton, A. Gillespie, and K. Stempel. 1972. Metabolic profiles of three fiber types of skeletal muscle in guinea pigs and rabbits. Biochemistry. 11:2627-2633.

15. Baldwin, K. M., W. G. Cheadle, O. M. Martinez, and D. A. Cooke. 1977. Effect of functional overload on enzyme levels in different types of skeletal muscle. J. Appl. Physiol. Respir. Environ. Exercise Physiol. 42:312-317.

16. Mansour, T. E., N. Wakid, and H. M. Sprouse. 1966. Studies on heart phosphofructokinase: purification, crystallization and properties of sheep heart phosphofructokinase. J. Biol. Chem. 241:1512-1521.

17. Srere, P. A. 1969. Citrate synthase. Methods Enzymol. 13:3-5.

18. Steel, R. G. D., and J. H. Torrie. 1960. Principles and Procedures of Statistics with Special Reference to the Biological Sciences. McGraw-Hill Book Co., New York. 67-86.

19. Kelsen, S. G., T. Wolanski, G. S. Supinski, and Y. Roesshann. 1983. The effect of elastase-induced emphysema on diaphragmatic muscle structure in hamsters. Am. Rev. Respir. Dis. 127:330-334.

20. Riley, D. A., and A. J. Berger. 1979. A regional histochemical and electromyographical analysis of the cat respiratory diaphragm. Exp. Neurol. 66:636-649. 
21. Keens, T. A., V. Chen, P. Patel, P. O'Brien, H. Levison, and C. D. Ianuzzo. 1978. Cellular adaptations of the ventilatory muscles to a chronic increased respiratory load. J. Appl. Physiol. 44:905-908.

22. Park, S. S., I. P. Goldring, C: S. Shim, and M. H. Williams. 1969. Mechanical properties of the lung in experimental pulmonary emphysema. J. Appl. Physiol. 26:738-744.

23. Faulker, J. A., L. C. Maxwell, D. A. Brook, and D. A Lieberman. 1971. Adaptation of guinea pig plantaris muscle fibers to endurance training. Am. J. Physiol. 221:291-297.

24. Farkas, G. A., and Ch. Roussos. 1983. The diaphragm in emphysematous hamsters: sarcomere adaptability. J. Appl. Physiol. 54:1635-1640.

25. Ishikawa, S., and J. A. Hayes. 1973. Functional morphometry of the diaphragm in patients with chronic lung disease. Am. Rev. Respir. Dis. 108:135-138.

26. Scott, K. W. M., and J. Hoy. 1976. The cross sectional area of diaphragmatic muscle fibers in emphysema, measured by an automated image analysis system. J. Pathol. 120:121-128.

27. Sanchez, J., J. P. Derenne, B. Debesse, M. Riquet, and H. Monod. 1982. Typology of the respiratory muscles in normal men and in patients with moderate chronic respiratory disease. Bull. Eur. Physiopathol. Respir. 18:901-914.

28. Hughes, R. L., H. Katz, V. Sahgal, J. A. Campbell, R. Hartz, and T. W. Shields. 1983. Fiber size and energy metabolites in five separate muscles from patients with chronic obstructive lung disease. Respiration. 44:321-328.

29. Campbell, J. A., R. L. Hughes, V. Sahgal, J. Frederiksen, and T. W. Shields. 1980. Alterations in intercostal muscle morphology and biochemistry in patients with obstructive lung disease. Am. Rev. Respir. Dis. 122:679-686.

30. Dudley; G. A., W. M. Abraham, and R. L. Terjung. 1982. Influence of exercise intensity and duration on biochemical adaptations in skeletal muscle. J. Appl. Physiol. Respir. Environ. Exercise Physiol. 53:844-850.

31. Minh, V. D., G. F. Dolan, R. F. Konopka, and K. M. Moser. 1976. Effect of hyperinflation on inspiratory function of the diaphragm J. Appl. Physiol. 40:67-73.

32. Baldwin, K. M., W. W. Winder, R. L. Terjong, and J. O. Holloszy. 1973. Glycolytic enzymes in different types of skeletal muscle: adaptation to exercise. Am. J. Physiol. 225:962-966.

33. Goldspink, G., and P. S. Ward. 1979. Changes in rodent muscle fibre types during post-natal growth, undernutrition and exercise. J. Physiol. 296:453-469.

34. Knowlton, G. C., and H. M. Hines. 1939/40. The effects of growth and atrophy upon the strength of skeletal muscle. Am. J. Physiol. 128:521-525.

35. Sharp, J. T., P. Van Lith, C. Veznuchprayoon, R. Brwey, and F. N. Johnson. 1968. The thorax in chronic obstructive lung disease. Am. J. Med. 44:39-46. 